\title{
Development And Early Feasibility Testing Of A Mind-Body Physical Activity Program For Patients With Heterogeneous Chronic Pain; The GetActive Study
}

This article was published in the following Dove Press journal: Journal of Pain Research

\author{
Jonathan Greenberg ${ }^{1,2}$ \\ Ann Lin' \\ Emily L Zale' \\ Ronald J Kulich (iD) 2,3 \\ Peter James ${ }^{2,4}$ \\ Rachel A Millstein (1) ${ }^{1,2}$ \\ Hannah Shapiro $\mathbb{D}^{5}$ \\ Michael E Schatman (iD ${ }^{6,7}$ \\ Robert R Edwards ${ }^{2,8}$ \\ Ana-Maria Vranceanu ${ }^{1,2}$ \\ 'Integrated Brain Health Clinical and \\ Research Program, Massachusetts \\ General Hospital, Boston, MA, USA; \\ ${ }^{2}$ Harvard Medical School, Boston, MA, \\ USA; ${ }^{3}$ Center For Pain Medicine, \\ Massachusetts General Hospital, Boston, \\ MA, USA; ${ }^{4}$ Department of Population \\ Medicine, Harvard T. H. Chan School of \\ Public Health, Boston, MA, USA; \\ ${ }^{5}$ Department of Biology, Tufts University, \\ Medford, MA, USA; ${ }^{6}$ Research and \\ Network Development, Boston Pain \\ Care, Waltham, MA, USA; ${ }^{7}$ Department \\ of Public Health and Community \\ Medicine, Tufts University School of \\ Medicine, Boston, MA, USA; ${ }^{8}$ Pain \\ Management Center, Brigham and \\ Women's Hospital, Boston, MA, USA
}

Correspondence: Ana-Maria Vranceanu Integrated Brain Health Clinical and Research Program, Department of Psychiatry, Massachusetts General Hospital/Harvard Medical School, One

Bowdoin Square, $I^{\text {st }}$ Floor, Suite 100 ,

Boston, MA, USA

Tel + I 6177244977

Fax + | 617-643-8777

Email avranceanu@mgh.harvard.edu
Background: Increasing physical function is a challenging, yet imperative goal of pain management programs. Physical activity can improve physical function, but uptake is low due to chronic pain misconceptions, poor pain management skills, and doing too much too soon.

Purpose: To increase physical function by 1) adapting an evidence-based, group, mind-body program to address the needs of patients with heterogeneous chronic pain and to facilitate individually tailored quota-based pacing with a Fitbit (GetActive with Fitbit) or without it (GetActive) (phase 1), and 2) assessing preliminary feasibility benchmarks (phase 2).

Methods: We followed evidence based frameworks for developing interventions and for early feasibility testing. In phase 1 we conducted 4 focus groups with 22 patients with heterogeneous chronic pain and adapted the mind-body program. In phase 2 we conducted a nonrandomized pilot trial of the 2 programs $(\mathrm{N}=7$ and 6) with qualitative exit interviews.

Results: Focus groups showed high interest in increasing activity, a preference for walking linked to pleasurable activities, using a Fitbit to track number of steps, and learning skills to manage pain and aid with increased activity. Both programs had good to excellent feasibility markers. Participation in both programs was associated with signal of improvements in physical and emotional function, as well as intervention targets. Exit interviews confirmed high satisfaction and suggested modification.

Conclusion: Results informed subsequent adaptations of the 2 programs and methodology for an ongoing pilot randomized controlled trial (RCT) of the 2 programs, necessary before an efficacy RCT of the 2 programs against an education control.

Keywords: mind-body, chronic pain, fitbit, physical activity, focus groups, feasibility, physical function

\section{Introduction}

Chronic pain, defined as persistent pain that lasts more than $3-6$ months, ${ }^{1}$ is prevalent, ${ }^{2}$ costly, ${ }^{2}$ and associated with decreased physical and emotional function. ${ }^{3}$ Over the last decade, mind-body (e.g. meditation and mindful-movement based) programs have been increasingly popular among patients with chronic pain, and have been shown to lead to improvements in anxiety, depression, and acceptance of symptoms. $^{4}$ However, effect sizes for improvements in self-reported emotional ${ }^{4-6}$ and physical functioning ${ }^{6-8}$ have been small to moderate, and tended to dissipate 
over time. ${ }^{4-6,8}$ Existent mind-body interventions do not incorporate systematic gains in physical activity despite its positive effects on emotional and physical function. Thus, there is a need for novel interventions that incorporate mind-body and physical activity skills in order to sustainably and substantially improve emotional and physical function among patients with chronic pain. The importance of comprehensive assessment of physical function has recently been specifically emphasized. The Recent Initiative on Methods, Measurement, and Pain Assessment in Clinical Trials (IMMPACT) ${ }^{9}$ and International Classification of Functioning, Disability and Health $(\mathrm{ICF})^{10}$ guidelines, as well as a recent review of mind-body interventions for chronic pain ${ }^{11}$ clearly specify that physical function should be comprehensively assessed by combining self-report, performance-based (e.g., walk test), and objective (e.g., accelerometer) measures in order to better understand effects of interventions and also improve overall efficacy and effect sizes. However, no chronic pain clinical trials to date comply. ${ }^{11}$ There is therefore a need for comprehensive assessments of physical function among patients with chronic pain.

Physical activity is an important target for patients with chronic pain, who tend to be sedentary, deconditioned, and thus at risk for further pain conditions and disability. ${ }^{12}$ Moreover, such sedentariness negatively impacts patients' mood, motivation, emotional function, and mental health. ${ }^{13,14}$ Walking - the preferred physical activity modality in chronic pain patients ${ }^{15}$ - is efficacious in improving self-reported function, particularly when quota-based (i.e. when activity is dependent on a given quota rather than dependent on pain levels ${ }^{16-18}$ ), although uptake and adherence are problematic. ${ }^{12,19}$ Activity monitoring devices such as Fitbit provide the user with feedback about their level activity and progress, and present an opportunity to actively reinforce the patient in incremental quota-based gains tailored to each patient's ability, thereby facilitating physical activity. ${ }^{20}$ Such devices are showing promising use in patients with chronic illness, ${ }^{21,22}$ including initial reports of effectiveness among patients with chronic pain. ${ }^{23,24}$ Our guiding hypothesis is that the most effective and efficient way to sustainably increase emotional and physical function among chronic pain patients is by 1) gradual and quota contingent increases in activity (walking) paired with re-engagement in activities of daily living; 2) monitoring and reinforcing quota contingent activity goals (number of steps) with a digital activity monitoring device (Fitbit)), and 3) teaching mind-body skills to manage pain, and target barriers to engaging in physical activity.
The goal of this paper is to set the stage for a future fully powered efficacy RCT to test our guiding hypothesis. Here, we report on a 2-phased study informed by the National Institute of Health (NIH) stage model for behavioral intervention development, ${ }^{25}$ National Institute of Complementary and Integrative Health (NCCIH) model for developing and testing mind-body intervention, ${ }^{26}$ and recent recommendations for early feasibility testing. ${ }^{27,28}$ In phase 1, we conducted focus groups to with the aim of informing adaptation of the 8-session mind-body Relaxation Response Resiliency Program (3RP) ${ }^{29}$ and developing the GetActive and GetActive with Fitbit programs, including treatment manuals and study procedures. The 3RP program was chosen specifically due to its demonstrated efficacy ${ }^{30}$ and its multimodal structure (e.g. including relaxation response, coping, CBT, and positive psychology skills), which has significant benefits over unimodal programs. ${ }^{31}$ In phase 2 , we conducted a nonrandomized pilot trial with exit interviews of the GetActive and GetActive with Fitbit programs with the aim of exploring feasibility markers and within group improvements on physical function and other relevant outcomes, to inform additional modifications before future phases of the study - phase 3 (RCT of GetActive versus GetActive with Fitbit) and phase 4 (efficacy RCT of GetActive versus GetActive with Fitbit versus health education control).

\section{Phase I: Development Of The GetActive And GetActive With Fitbit Programs Methods}

First, our multidisciplinary team with expertise in chronic pain, mind-body medicine, and physical activity modified the 3RP manual ${ }^{29}$ to target the needs of patients with chronic pain. ${ }^{32,33}$ The 3RP teaches relaxation response (e.g., diaphragmatic breathing, mindfulness), stress awareness and coping (e.g., adaptive thinking, acceptance), and positive psychology (e.g., humor, optimism) skills. We also proposed new pain specific skills including a discussion on the rationale and practice of quota-based, non pain contingent increases in activity using time goals (GetActive) and step counts (GetActive with Fitbit). Further we added behavioral activation techniques (e.g. identifying and scheduling physical activities that are achievable for the patient; targeting motivation ${ }^{34}$ ) and paired increased activity with reengagement in activities 
of daily living (e.g. increasing step count via activities such as walking to the grocery store or commuting home). Next, we developed a semi-structured qualitative interview script to gauge participants' views on the challenges of living with chronic pain, perception of the proposed modifications of the 3RP skills, perception of the additional proposed skills targeting pain, physical activity, emotional and physical functioning, and barriers and facilitators for Fitbit use and program participation.

\section{Participants And Recruitment}

Twenty-two adults with chronic pain participated in four focus groups $(\mathrm{N}=2-9$ each). Participants were recruited between September 2017 and January 2018, primarily via the Pain Clinic at the Massachusetts General Hospital, through direct referrals from study staff, and through flyers distributed through a hospital-wide email advertising clinical research. Interested participants were screened over the phone by a trained research assistant. Inclusion criteria were: 1) age $\geq 18$;2) nonmalignant musculoskeletal pain for more than 3 months; 3 ) able to perform a 6 min walk test; ${ }^{35}$ 4) own a smartphone with Bluetooth 4.0 or computer; 5) on a stable dose of psychotropic or pain medication and willing to maintain a stable dose. Exclusion criteria were: 1) medical illness expected to worsen in the next 6 months; 2) serious psychiatric illness (untreated schizophrenia, active suicidality); 3) current substance use disorder; 4) practice of yoga/meditation once per week for 45 mins or more within the last 3 months; and 5) regular physical exercise for $>30$ min daily. The latter two exclusion criteria were chosen to ensure that participants interviewed would be similar to those who will participate in the intervention, thus eliminating confounds. All screenings were reviewed by a clinical psychologist. Of the 86 participants screened, 39 did not meet study criteria, 16 had scheduling difficulties, and 9 dropped prior to enrollment. All participants provided written informed consent prior to the focus groups. All study procedures were approved by the Institutional Review Board of Massachusetts General Hospital, and all procedures were in compliance with the Declaration of Helsinki. Study procedures are further detailed in Figure 1.

\section{Focus Groups}

The interview focused on 4 major areas: 1) challenges of living with chronic pain, 2) perception of modified 3RP specific skills to address needs of patients with chronic pain, 3) perception of additional skills targeting activity, emotional and physical functioning, and 4) barriers/
Phase I Procedure

\section{Referrals from clinicians and partners clinical trials}

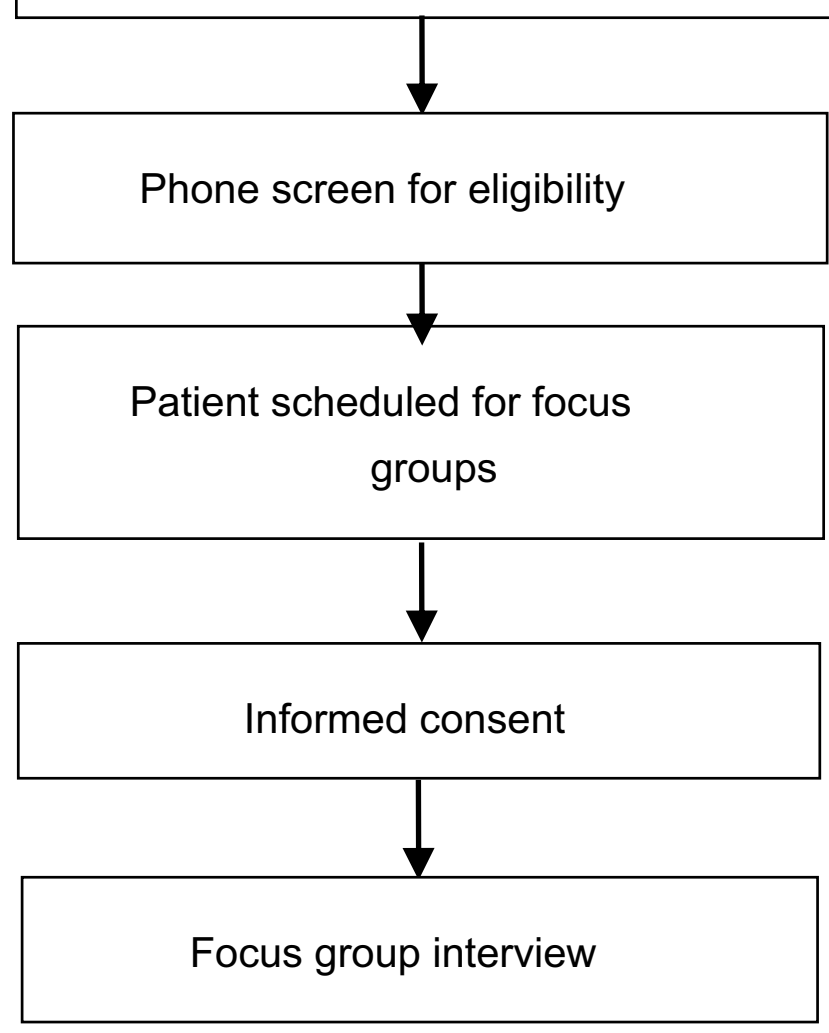

Figure I Phase I procedure.

facilitators for Fitbit use and program participation. Interviews lasted 40-60 mins depending on the size of each group and were audio-recorded. Groups were led by clinical health psychologists with expertise in treating individuals with chronic pain. At the end of each group participants completed a demographic questionnaire (Table 1). Each participant was reimbursed $\$ 20$ for participation.

\section{Analysis Plan}

We used guidelines for qualitative data analyses that focused on a framework approach with the goal of minimizing bias. ${ }^{36,37}$ The framework approach is a method of qualitative data analysis which involves the indexing of thematic content into categories, which are then used to compare and contrast the content and draw descriptive 
Table I Demographic And Clinical Patient Characteristics Of Participants In Focus Groups And Open Pilot Groups

\begin{tabular}{|c|c|c|c|}
\hline & Focus Groups n=22 & Open Pilot: GetActive $n=6$ & $\begin{array}{l}\text { Open Pilot: GetActive } \\
\text { With Fitbit } n=7\end{array}$ \\
\hline & $M(S D)$ & $M(S D)$ & $M(S D)$ \\
\hline Age & $49.7(17.8)$ & $46.7(15.0)$ & $41.6(14.5)$ \\
\hline Overall pain (scale $\mathrm{I}-10$ ) & $7.3(2.1)$ & - & - \\
\hline Pain at rest (scale $1-10$ ) & - & $5.2(2.2)$ & $5.3(2.3)$ \\
\hline Pain with activity (scale I-10) & - & $6.7(1.5)$ & $7.6(1.8)$ \\
\hline & $\mathbf{N}(\%)$ & $\mathbf{N}(\%)$ & $\mathbf{N}(\%)$ \\
\hline $\begin{array}{l}\text { Gender } \\
\text { Male } \\
\text { Female }\end{array}$ & $\begin{array}{l}9(40.9 \%) \\
13(59.1 \%)\end{array}$ & $\begin{array}{l}2(33.3 \%) \\
4(66.7 \%)\end{array}$ & $\begin{array}{l}\text { I (I4.3) } \\
6(85.7 \%)\end{array}$ \\
\hline $\begin{array}{l}\text { Ethnicity } \\
\text { Hispanic or Latino/Latina } \\
\text { Not Hispanic or Latino/Latina }\end{array}$ & $\begin{array}{l}5(22.7 \%) \\
17(77.3 \%)\end{array}$ & $\begin{array}{l}3(50.0 \%) \\
3(50.0 \%)\end{array}$ & $\begin{array}{l}\text { I (14.3\%) } \\
6 \text { (85.7\%) }\end{array}$ \\
\hline $\begin{array}{l}\text { Race } \\
\text { American Indian/Alaskan Native } \\
\text { Asian } \\
\text { Black/African American } \\
\text { Native Hawaiian/Pacific Islander } \\
\text { White } \\
\text { More Than One Race } \\
\text { Choose Not to Answer }\end{array}$ & $\begin{array}{l}\text { I }(4.5 \%) \\
2(9.1 \%) \\
0(0.0 \%) \\
0(0.0 \%) \\
14(63.6 \%) \\
2(9.1 \%) \\
3(13.6 \%)\end{array}$ & $\begin{array}{l}0(0.0 \%) \\
2(33.3 \%) \\
0(0.0 \%) \\
0(0.0 \%) \\
2(33.3 \%) \\
2(33.3 \%) \\
0(0.0 \%)\end{array}$ & $\begin{array}{l}0(0.0 \%) \\
\text { I }(14.3 \%) \\
\text { I }(14.3 \%) \\
0(0.0 \%) \\
4(57.1 \%) \\
\text { I }(14.3 \%) \\
0(0.0 \%)\end{array}$ \\
\hline $\begin{array}{l}\text { Marital Status } \\
\text { Single, never married } \\
\text { Living with someone } \\
\text { Married } \\
\text { Divorced } \\
\text { Separated } \\
\text { Widowed }\end{array}$ & $\begin{array}{l}4(18.2 \%) \\
4(18.2 \%) \\
10(45.5 \%) \\
2(9.1 \%) \\
1(4.5 \%) \\
1(4.5 \%)\end{array}$ & $\begin{array}{l}2(33.3 \%) \\
0(0.0 \%) \\
2(33.3 \%) \\
2(33.3 \%) \\
0(0.0 \%) \\
0(0.0 \%)\end{array}$ & $\begin{array}{l}4(57.1 \%) \\
0(0.0 \%) \\
2(28.6 \%) \\
1(14.3 \%) \\
0(0.0 \%) \\
0(0.0 \%)\end{array}$ \\
\hline $\begin{array}{l}\text { Education } \\
\text { High school ( } 12 \text { years) } \\
\text { Some college/Associates degree (<16 years) } \\
\text { Completed college ( } 16 \text { years) } \\
\text { Graduate/professional degree ( }>16 \text { years) }\end{array}$ & $\begin{array}{l}0(0.0 \%) \\
12(54.5 \%) \\
2(9.1 \%) \\
8(36.4 \%)\end{array}$ & $\begin{array}{l}\text { I }(16.7 \%) \\
0(0.0 \%) \\
\text { I (16.7\%) } \\
4(66.7 \%)\end{array}$ & $\begin{array}{l}2(28.6 \%) \\
2(28.6 \%) \\
2(28.6 \%) \\
I(14.3 \%)\end{array}$ \\
\hline
\end{tabular}

conclusions. ${ }^{38}$ Study staff transcribed all recordings which were subsequently independently checked by an additional staff member. Next, we identified themes and developed a coding structure. Two additional staff members not involve in the development of the coding structure independently coded the data using NVivo $11 .{ }^{39}$ Whenever the data did not fit an existing code, new ones were added. Discrepancies were resolved through consensus between the coders and feedback from study staff with expertise in qualitative methods. A kappa coefficient was calculated to determine coding precision.

\section{Results}

The Kappa index for coding was high (0.76), indicating high consensus between raters. Coding yielded 4 major themes (bolded) and 9 subthemes (italicized). Table 2 provides further details about the themes and subsequent adaptations to the program manual. 


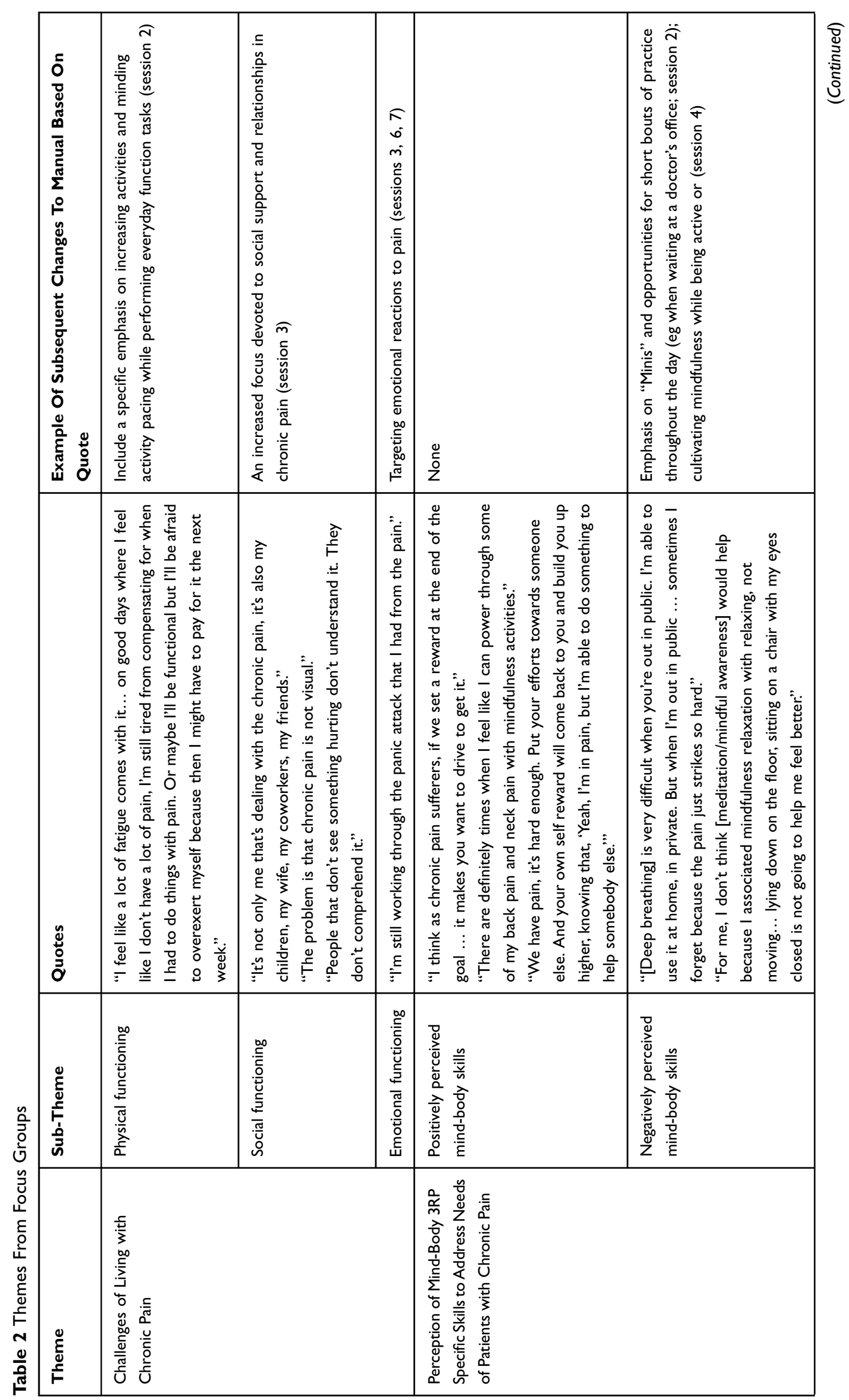




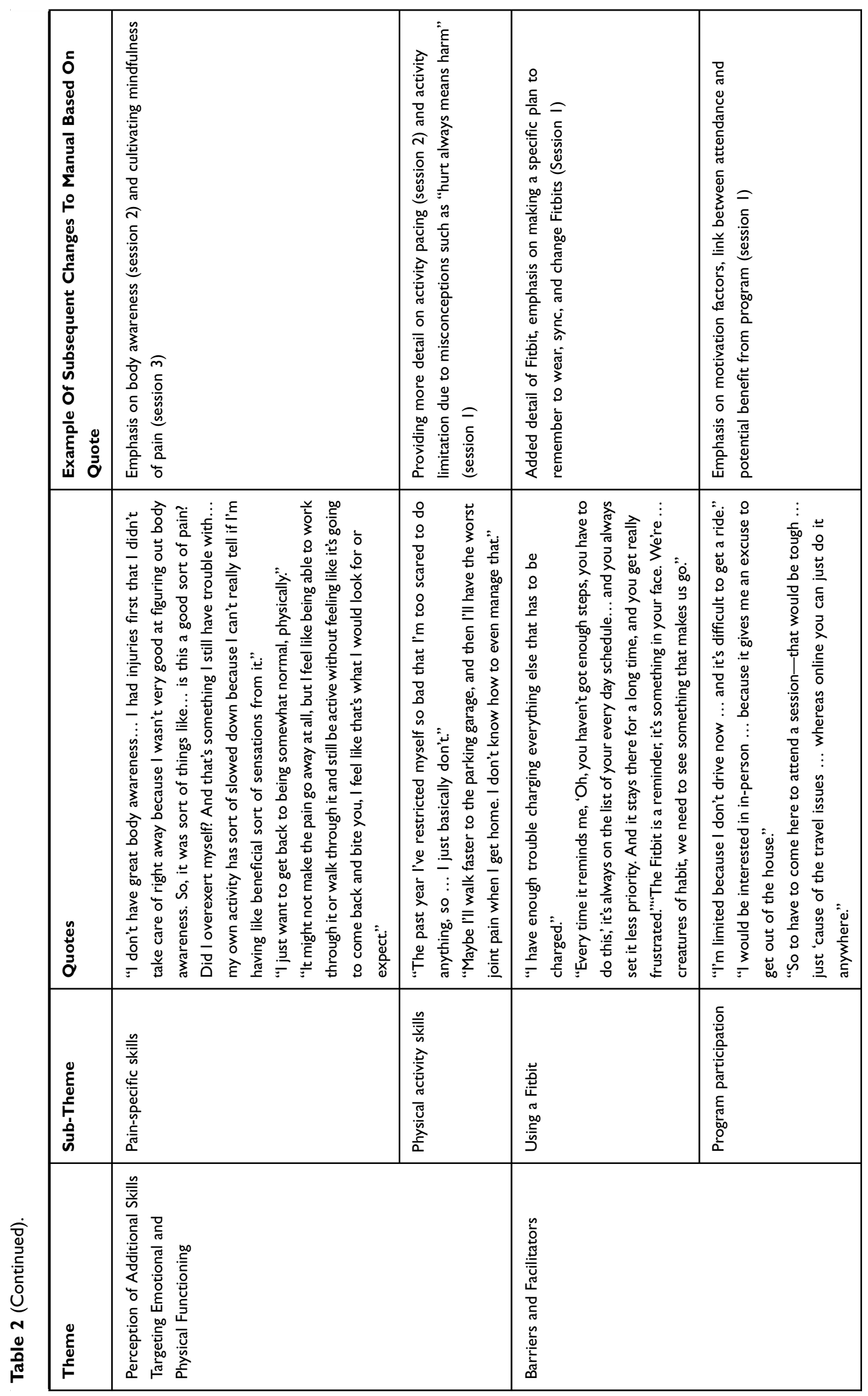




\section{Theme \#I: Challenges Of Living With Chronic Pain}

Social functioning. Most participants reported that their chronic pain affects their loved ones and impairs their own social functioning. They felt disconnected and misunderstood by others, who may not be aware of their pain. Participants expressed a wish to re-strengthen and restore their relationships.

Physical functioning. Participants noted inactivity/significant reduction in activities due to pain and associated physical symptoms such as exhaustion. They reported that their chronic pain significantly interferes with their ability to keep a job, engage with family or friends, and impacts their performance at work due to impaired concentration or frequent absenteeism.

Emotional functioning. Many participants reported feeling overwhelmed, anxious, and depressed due to pain. They also felt frustrated about the medical system and noted that their pain management needs are unmet (e.g., insurance companies not covering their preferred treatments, medical providers unable or unwilling to offer useful treatment, being told to "get over it," and being dismissed from care). Some participants reported feeling that their doctors invalidate their experiences, are unable to understand them, or set unrealistic expectations.

\section{Theme \#2: Perception Of The Modified 3RP Specific Skills To Address Needs Of Patients With Chronic Pain}

The majority of participants liked the modified 3RP skills. Participants appreciated the provided rationale for using these skills to improve pain management and reported their interest in learning these skills themselves. Several participants noted that they had been or were currently intermittently using some of the 3RP strategies, such as gratitude, humor, and optimism, as well as practices such as deep breathing and meditation. They noted these skills as being helpful and associated with improvement in pain, relaxation, and motivation. However, many noted challenges adhering to these skills (e.g. finding time to practice) and reported their belief that the group setting would help with motivation. They were receptive to pain specific meditations and learning to use these skills consistently to manage pain. Other skills, such as goal setting, were also perceived as helpful means to increase motivation and adherence over time. A minority of participants, however, expressed some reluctance, as they associated mind-body skills with inactivity or laziness. Other participants shared concerns about the logistics of practicing these skills in public.

\section{Theme \#3: Perception Of Additional Skills Targeting Emotional And Physical Functioning}

Pain specific skills. Participants indicated that understanding the disability spiral, myths about pain (e.g., harm versus hurt), learning quota-based (non-pain contingent) pacing, the use of metaphors such as "the true pain alarm" (i.e. when pain signifies damage) vs the "false pain alarm" (i.e. when it does not) and setting specific goals toward increasing activity can all be useful tools to help manage their pain and become more physically active regardless of pain. Participants reported that they expected these new skills to better enable them to tend to their day-to-day activities despite the pain, rather than eliminate their pain altogether.

Physical activity skills. Generally, participants reported being sedentary, and indicated fear of exacerbating pain as a primary motive for avoiding activity. They reported continuously restricting their activities and the heavy toll associated with these restrictions. Several participants attempted to be active, primarily through walking, but noted immediate discouragement due to increased pain. All participants noted increased activity during the "good days," and then needing to rest the following day(s) because of activity-related pain. Despite these challenges, most participants preferred walking as a primary mode of physical activity, although a minority of participants reported interest in other activities, such as aquatic exercise. The provided rationale for using pacing and quotabased, non-pain contingent increases in walking paired with re-engagement in daily activities made sense to participants.

\section{Theme \# 4: Barriers And Facilitators}

Using a Fitbit. Participants noted an understanding of the rationale behind using a Fitbit to help them gradually increase their daily steps while learning to pace themselves. They described their desire to meet a predetermined step goal, and to develop a habit of wearing the Fitbit and incorporate it into their normal routines. Participants noted two barriers to engaging with the Fitbit: forgetting to charge it and forgetting to wear it. They noted that having a concrete plan for when to charge their Fitbit (e.g., while taking a shower) as well as reminders to wear it would help adherence. They also agreed that receiving reminder phone calls from study staff and checking their progress on their phones would help increase both adherence and motivation. 
Program participation. Participants identified several barriers to participation, including burden of travel to the clinic, challenges finding parking, cost of parking, distance of clinic to the closest train station, and scheduling conflicts. Several participants noted an increased challenge with both attendance and activity during the winter months in New England. Participants also identified several facilitators to program participation, such as the interactive nature of the group setting, the opportunity to leave their houses, and optimism regarding the program's potential to help them better manage their pain and increase their ability to engage in activities that they enjoyed prior to the onset of their chronic pain.

\section{Phase 2: Open Pilot Studies Methods \\ Participants And Recruitment}

Recruitment took place between January and April 2018 via the same methods described in Phase 1. Inclusion criteria were identical to those of phase 1 , with an additional criterion requiring the ability to participate in 8 in-person group treatment sessions. We also excluded participants who used a Fitbit tracker in the past 3 months. A total of 13 participants enrolled (Figure 2 and Table 1). The first six participated in the GetActive program and the next seven in the GetActive with Fitbit program. All participants provided written informed consent prior to participation. All study procedures were approved by the Institutional Review Board of Massachusetts General Hospital, and all procedures were in compliance with the Declaration of Helsinki. Study procedures are further detailed in Figure 3.

\section{Procedure For GetActive And GetActive With Fitbit}

A research assistant scheduled the consent and assessments for all participants. A clinician described the study in detail and performed consent procedures. After consent was provided, participants completed study questionnaires in person, as a group. A research assistant was available to answer questions and check that all questions were answered. The research assistant also performed the 6 min walk test. ${ }^{35}$ At the end of the $2 \mathrm{hr}$ assessment session, participants received a wGT3X-BT ActiGraph accelerometer (Pensacola, FL, USA) to wear daily for 1 week, wear instructions (including no changes in routine activities), and were asked to keep a wear time log. The research assistant called participants daily with reminders to wear the ActiGraphs and help problem solve issues in real time. Participants returned the ActiGraphs and the logs the following week, which coincided with Session 1 of the group program. At the end of the program (week 8), participants were asked to wear the ActiGraphs again for one week, following the same guidelines previously described. They returned for the final assessment session a week later, during which they completed the post intervention questionnaires, the $6 \mathrm{~min}$ walk test, and exit interviews.

\section{Additional Procedural Steps For GetActive With Fitbit} The research assistant downloaded the ActiGraph data while the study clinician delivered the first group session, and prepared individual Fitbit accounts with individual steps counts. Step goals for each participant were determined based on predefined criteria (see GetActive and GetActive with Fitbit programs section below). At the end of the GetActive with Fitbit group session, the research assistant paired each participant's phone with the Fitbit. During each of the subsequent group visits, the research assistant manually programed steps goals directly into each participant's Fitbit using the same criteria.

\section{GetActive And GetActive With Fitbit Programs}

The two 8-session weekly programs retained the core components of the $3 \mathrm{RP}^{29}{ }^{29}$ modified for pain: 1) elicitation of the relaxation response (RR) through general, pain and activityspecific meditations; 2) reducing overall reactivity to stress and pain through adaptive thinking and acceptance-based techniques, and 3) increasing connectedness to self and others generally and when in pain through social support and positive psychology skills, as well as the emphasis on home practice (adaptive goal setting: "SMART goals"; defined as goals that are specific, measurable, attainable, relevant, and time-based"), ${ }^{29}$ daily $\mathrm{RR}$ practice and appreciations.

We also added 5 components: 1) education regarding myths about chronic pain and activity to help participants challenge their ingrained misconceptions regarding pain and activity 2) the "true versus false pain alarm skill," to help participants understand the difference between harmful pain (e.g., acute pain, new injury), and non-harmful pain (e.g., chronic pain which is not indicative of current physical damage); 3) quota-based, non-pain contingent activity pacing, to help them gradually increase activity; 4) behavioral activation (e.g., reengagement in meaningful activities of daily living) paired with quota based pacing based increases in activity; 5) challenging misconceptions around mindfulness meditation (Table 3). 


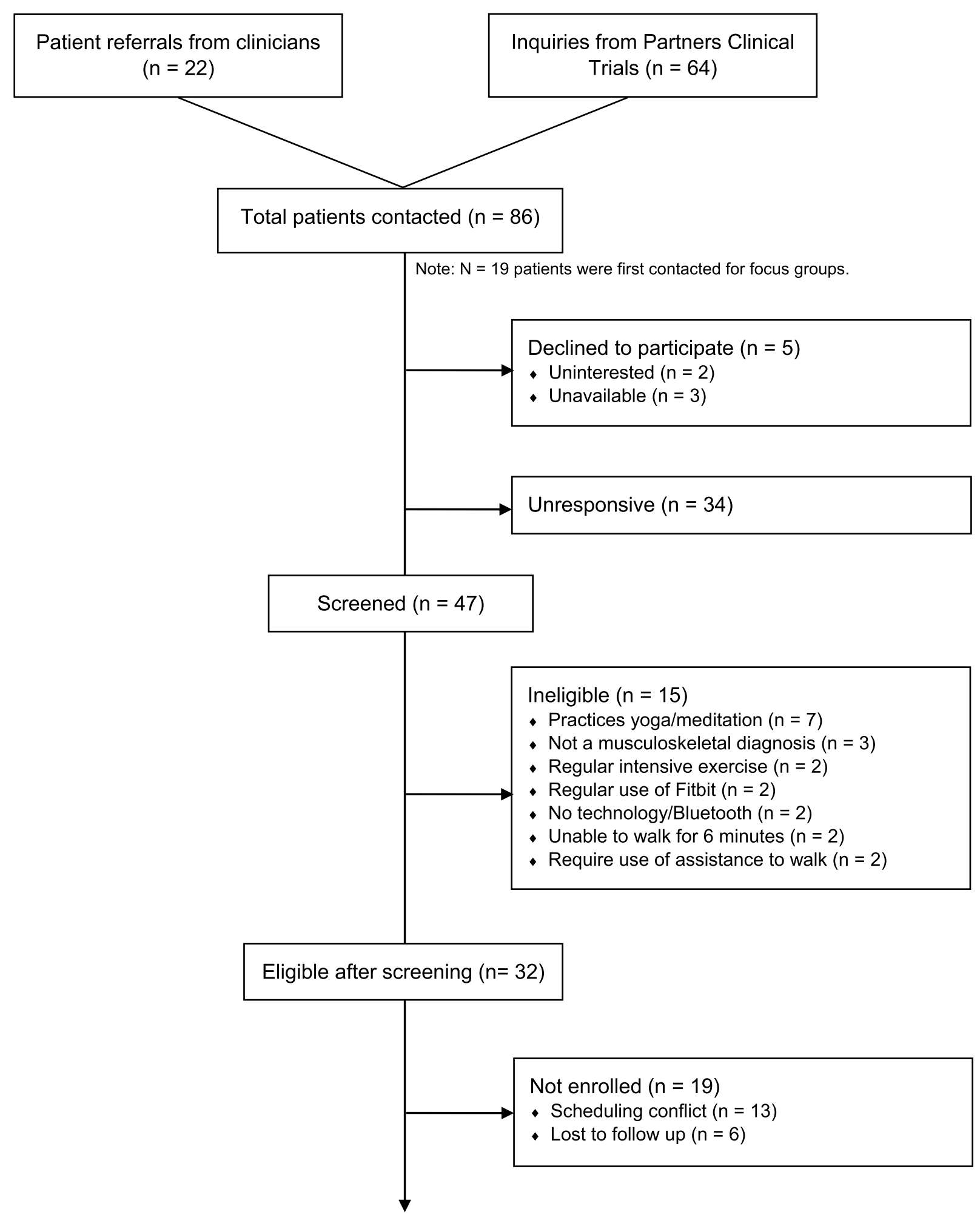

Enrolled $(n=13)$

Figure 2 Participant flow; Phase 2. 


\section{Phase II Procedure}

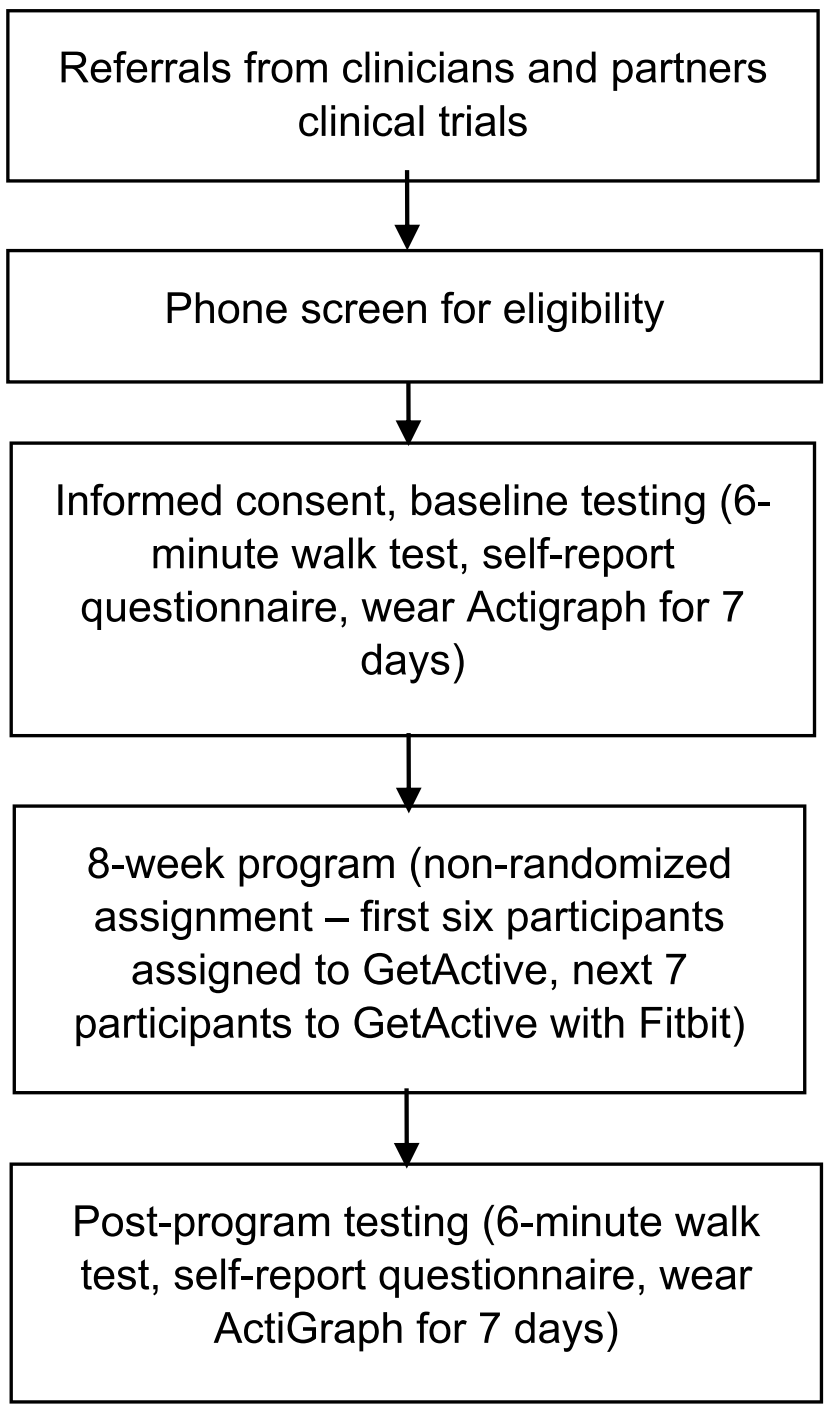

Figure 3 Phase 2 procedure.

For the GetActive with Fitbit group, we used the Fitbit's step-count to assess weekly physical activity, determine participants' pacing plans, and set weekly activity SMART goals. Each week, steps were assessed over 5day blocks. After the ActiGraph-monitored baseline stepcount was determined, participants set this count as their initial step goal, which was uploaded onto the Fitbit. Participants had to reach their goal, on average, by the day of the session. We excluded the highest and lowest step-count days. Patients were allowed 2 "rest days" per week. Missed samples or days on which patients forgot to charge or wear their devices were counted as rest days. If participants met the 5-day goal criterion, they were given the option of repeating their step goal the following week or increasing their step goal by $10 \%$. Participants paired their Fitbits with their smartphones, which automatically downloaded data on a daily basis. A staff member calculated patients' upcoming step goals and provided the study clinician with individual adherence data for each participant, used when discussing homework adherence and to set homework including new step goals. In the GetActive program, participants' physical activity levels were assessed via activity logs. We calculated their corresponding pacing plans and SMART goals based on time spent being physically active or distance walked, based on individual patient preference. 
Table 3 GetActive With Fitbit Session Topics And Skills

\begin{tabular}{|c|c|c|}
\hline Session & Topic & Skills \\
\hline I & $\begin{array}{l}\text { Pain Management, Stress Management, } \\
\text { and Resiliency Training }\end{array}$ & $\begin{array}{l}\text { Pain myths; body awareness; setting activity SMART goals; quota-based activity pacing; using a } \\
\text { Fitbit (for GetActive with Fitbit group) }\end{array}$ \\
\hline 2 & Relaxation Skills to Manage the Pain Alarm & $\begin{array}{l}\text { Activity barriers; adherence to Fitbit; pairing steps with activities of daily living; relaxation vs } \\
\text { stress response; deep breathing; sleep; meditation }\end{array}$ \\
\hline 3 & $\begin{array}{l}\text { Stress and Symptom Awareness for } \\
\text { Chronic Pain Patient }\end{array}$ & Mindful awareness; stress warning signals; social support; the pain cycle \\
\hline 4 & Mending the Chronic Pain Mind and Body & Pairing Activity, with mind-body skills; negative automatic thoughts and adaptive thinking \\
\hline 5 & Creating an Adaptive Perspective & Guided imagery; healthy eating; "stop, breathe, reflect, choose" and links to chronic pain \\
\hline 6 & Promoting Positivity & $\begin{array}{l}\text { Loving kindness meditation; optimistic storytelling; relaxation signals; healthy habits and links } \\
\text { to chronic pain }\end{array}$ \\
\hline 7 & Healing States of Mind & $\begin{array}{l}\text { Problem solving and acceptance; empathy and compassion; contemplation and how they help } \\
\text { with pain management and activity. }\end{array}$ \\
\hline 8 & Humor, Empathy and Staying Resilient & Humor and laughter; staying resilient for pain management; overview of resiliency skills \\
\hline
\end{tabular}

\section{Feasibility And Acceptability Assessments}

Assessment milestones were set a priori and were based on guidelines for feasibility studies. ${ }^{40,41}$ Feasibility of recruitment was assessed by determining the proportion of contacted patients who agreed to participate. A feasibility rate of $80 \%$ was considered excellent and $70 \%$ was considered good. Program acceptability was calculated by reporting the proportion of participants who attended 6 out of 8 treatment sessions. Feasibility was considered excellent when at least $70 \%$ participants attended at least 6 of the 8 planned treatment sessions (75\%). Therapist adherence to the manual was determined via checklists. An adherence rate of $100 \%$ was considered excellent and $75 \%$ good. Feasibility of quantitative measures was deemed acceptable if no questionnaires were fully missing in more than $25 \%$ participants, and if reliability was higher than 0.70 . Adherence to homework and activity were assessed via number of logs turned in by participants over the course of the study, and via reaching SMART goals and frequency of reported RR practice and appreciations. Adherence was deemed excellent if participants had at least 3 to 4 out of 7 days of completed homework (RR practice, SMART goal activity, and appreciations) OR at least 5 out of 7 days for one of the 3 components, by at least $80 \%$ of participants. Adherence was deemed good if at least 3 to 4 out of 7 days of completed homework (RR practice, SMART goal activity and appreciations) OR at least 5 out of 7 days for one of the 3 components, by at least $70 \%$ participants. ActiGraph adherence was assessed via daily wear of ActiGraph accelerometer (minimum $7 \mathrm{hrs} /$ day) at baseline and post intervention assessments. Adherence was deemed excellent if data were successfully collected at least 5 out of 6 days a week ( $>7$ wear hours a day; excluding the days participants received and returned the ActiGraphs), from at least $80 \%$ of participants during the pre- and post-assessments. Adherence was deemed good if data were collected from at least $70 \%$ of participants. Fitbit adherence was assessed via daily wear of a charged device for the GetActive with Fitbit group. Adherence to Fitbit was deemed excellent if data were collected from at least 5 of 7 days from at least $80 \%$ of participants and good if collected from $70 \%$ of participants. Satisfaction with the program was assessed via the Client Satisfaction Questionnaire (CSQ-3) ${ }^{42}$ and was determined by the percentage of participants scoring above the scale midpoint ( $\geq 75 \%$ excellent; $\geq 70 \%$ good).

\section{Physical Function Assessments \\ Objective Physical Function/Physical Activity}

During baseline and post-program testing, all participants wore a wGT3X-BT ActiGraph accelerometer (Pensacola, FL, USA) for 7 days to determine baseline average number of daily steps. An increase of 1000 steps is considered a minimally clinical important difference (MCID). ${ }^{43}$

\section{Performance-Based Physical Function}

We used the 6 min walk test, ${ }^{44}$ which measures the distance participants walk for 6 mins. The MCID in walking distance is $54 \mathrm{~m}^{44}$ 


\section{Patient-Reported Physical Function}

We measured self-reported physical function with the Patient-Reported Outcomes Measurement Information System (PROMIS) Physical Function, v.1.2.8b ${ }^{45}$ which assesses one's ability to perform activities ranging from self-care (activities of daily living) to more complex activities requiring a combination of skills, often within a social context. The MCID for this measure is $8 .^{46} \mathrm{We}$ additionally used the World Health Organization Disability Assessment Schedule (WHODAS) $2.0,{ }^{47} \mathrm{a}$ 36 -item questionnaire assessing functional difficulties in various domains (e.g., understanding and communicating, getting around, self-care, getting along with people, life activities, and participation in society). Internal reliability in the current sample was good (Cronbach's alpha $=0.81-0.87)$ for the PROMIS and excellent $(0.95-$ 0.96) for the WHODAS. There is no MCID available for WHODAS. We used both measures in order to be able to compare their respective sensitivity in preparation for a future RCT.

\section{Self-Reported Physical Activity Scale For Individuals With Physical Disabilities (PASIPD) ${ }^{48}$}

A 13-item questionnaire which assesses engagement in leisure, household, and work activities of varying intensities for the past 7 days. This measure has established validity, with low-to-moderate Cronbach's alpha values (0.37-0.65) within each of its five factors due to the small number of items in each factor. ${ }^{48}$ There is no MCID available for this measure.

\section{Emotional Function}

We assessed emotional function with the PROMIS depression v1.0.8b $\mathrm{b}^{49}$ as well as the PROMIS anxiety, v1.08a. ${ }^{50}$ The MCID for PROMIS depression is 5.19 and for PROMIS anxiety is $4.28 .^{51}$ Internal reliability for both measures in the current sample was excellent (Cronbach's alpha $=0.87-$ 0.96 for depression, $0.93-0.96$ for anxiety).

\section{Other Patient-Reported Assessments}

While our main quantitative outcomes, which we will test in the future efficacy RCT are emotional and physical function, at this stage it is important to test other variables that assess constructs that are directly targeted by the program, which may be mediators in a future RCT and will inform about the mechanisms of improvement. We used the following additional measures (MCID indicated when available):
Pain. We used the Numerical Rating Scale (NRS); ${ }^{52,53}$ 11 -point Likert scale, with " 0 " representing no pain and " 10 " representing the worst pain possible, during rest and activity. A 1-point change is considered the MCID for patients with musculoskeletal pain. ${ }^{54}$

Social isolation was assessed via the PROMIS Social Isolation short form (4a), ${ }^{55}$ a 4 -item questionnaire assessing perceptions of being avoided, excluded, detached, disconnected from, or unknown by, others. Internal reliability (Cronbach's alpha) for this measure was 0.60-0.82.

Mindfulness was assessed by the Cognitive and Affective Mindfulness Scale- Revised (CAMS-R), ${ }^{56}$ a 12-item self-report measure of the frequency with which patients use mindfulness behaviors (e.g., noticing thoughts without judgement) in their daily lives. Items are scored on a 4-point Likert scale, with "0" representing "rarely/not at all" and " 3 " representing "almost always". Internal reliability (Cronbach's alpha) for this measure was 0.63-0.77.

Emotional support was assessed via the PROMIS emotional support (4a), ${ }^{57}$ a 4 -item questionnaire measuring perceived feelings of being cared for and valued as a person and having close relationships. This measure had excellent internal reliability in the current sample (Cronbach's alpha=0.94-0.97).

Coping. Coping was assessed using (1) the Pain Catastrophizing Scale (PCS), ${ }^{58}$ which assesses hopelessness, helplessness and rumination about pain; (2) the Tampa Kinesiophobia Scale (TSK), ${ }^{59}$ which assesses fear of pain and activities that cause pain, and the Measure of Current Status (MOCS), ${ }^{60}$ which assesses ability to engage in a series of healthy coping skills (e.g., relaxation, social support, adaptive thinking). Internal reliability was found to be excellent (Cronbach's alpha $=0.93-0.94$ ) for the PCS, good (0.77-0.87) for the TSK, and acceptable (0.71-0.77) for the MOCS.

Perceived Improvement was assessed with the Modified Patient Global Impression of Change (MPGIC), ${ }^{61}$ in which participants assessed on a 7-point Likert scale (with 1 representing "very much improved") and "7" representing "very much worse" the extent to which they perceived that the intervention improved their pain, physical activity, physical function, emotional function, pain resiliency, and the degree to which the Fitbit helped to increase their activity (for the GetActive with Fitbit group). The rationale for using all of these measures concurrently is to help determine which the assessments best capture the effects of the program, and should be included in the future feasibility and efficacy RCTs. 


\section{Exit Interviews}

Following program completion, participants participated in semi-structured group-based exit interviews to assess their views on program components feasibility. All participants agreed to maintain confidentiality. Interviews lasted $\sim 60 \mathrm{mins}$ and were audio-recorded, with an emphasis on the importance of honest feedback to enable program optimization.

\section{Analysis Plan}

Consistent with common guidelines for feasibility studies, ${ }^{27}$ we did not test efficacy or perform between-group analyses. Rather, we limited our quantitative analyses to descriptive statistics for each quantitative measure, as well as withingroup pre-post comparisons using paired t-tests and Cohen's D effect sizes (ES) to broadly gauge for potential signals of improvement. To verify results of these comparisons due to the low sample size, we additionally conducted the non- parametric related-samples Wilcoxon signed rank test comparing pre and post values. Qualitative data analysis for the exit interviews followed the methods outlined above in Phase 1.

\section{Results}

\section{Feasibility And Acceptability Markers}

Feasibility of recruitment, credibility and expectancy, client satisfaction, acceptability of treatment, adherence to ActiGraphs and Fitbit, adherence to homework, therapist adherence to manual, and perception of improvement were good to excellent and similar for both groups (Table 4).

\section{Physical Function (Table 5)}

Objective physical function. Overall, both groups, when considered together, showed a significant increase in ActiGraph-measured number of steps from baseline to posttest (Figure 4). Participants in the GetActive with Fitbit group had a relatively high baseline step-count

Table 4 Feasibility And Acceptability Of The Open Pilot Studies

\begin{tabular}{|c|c|c|}
\hline Outcome & GetActive & GetActive With Fitbit \\
\hline Feasibility of recruitment & \multicolumn{2}{|c|}{47 participants out of 52 successfully contacted agreed to complete screening (excellent) } \\
\hline Credibility and expectancy & 5 out of 6 participants (83\%) scored over median split & $\begin{array}{l}5 \text { out of } 7 \text { participants }(7 \mid \%) \text { scored over } \\
\text { median split }\end{array}$ \\
\hline Client satisfaction & $\begin{array}{l}5 \text { out of } 6 \text { participants (83\%) scored over median split } \\
\text { (excellent) }\end{array}$ & $\begin{array}{l}6 \text { out of } 7 \text { participants ( } 86 \%) \text { scored over } \\
\text { median split (excellent) }\end{array}$ \\
\hline Acceptability of treatment & $\begin{array}{l}4 \text { out of } 6 \text { participants ( } 66 \% \text { ) attended } \geq 6 \text { out of } 8 \\
\text { sessions (acceptable) }\end{array}$ & $\begin{array}{l}6 \text { out of } 7 \text { participants ( } 86 \% \text { ) attended } \geq 6 \text { out } \\
\text { of } 8 \text { sessions (excellent) }\end{array}$ \\
\hline Adherence to ActiGraphs and Fitbit & $\begin{array}{l}6 \text { out of } 6 \text { participants at baseline ( } 100 \% \text { ) and } 5 \text { out of } 6 \\
\text { at post-test (83\%) recorded } \geq 5 \text { days of ActiGraph data } \\
\text { (excellent) }\end{array}$ & $\begin{array}{l}5 \text { out of } 7 \text { participants ( } 71 \% \text { ) recorded } \geq 5 \text { days } \\
\text { of ActiGraph data at both baseline and post- } \\
\text { test (good) } \\
6 \text { out of } 7 \text { participants }(86 \% \text { ) recorded at least } \\
5 \text { out of } 7 \text { days of Fitbit data every week } \\
\text { (excellent) } \\
\text { Participants met } 53 \% \text { of their step-count goals }\end{array}$ \\
\hline Adherence to homework & $\begin{array}{l}4 \text { out of } 6 \text { participants ( } 66 \% \text { ) completed } \geq 5 \text { out of } 7 \\
\text { weeks of homework (acceptable) }\end{array}$ & $\begin{array}{l}6 \text { out of } 7 \text { participants ( } 86 \% \text { ) completed } \geq 5 \\
\text { out of } 7 \text { weeks of homework (excellent) }\end{array}$ \\
\hline Therapist adherence to manual & $100 \%$ adherence (excellent) & $100 \%$ adherence (excellent) \\
\hline Feasibility of quantitative measures & No questionnaires missing fully (excellent) & No questionnaires missing fully (excellent) \\
\hline Patients' perception of improvement & $\begin{array}{l}5 \text { out of } 6 \text { participants (83\%) reported overall } \\
\text { improvement (excellent) }\end{array}$ & $\begin{array}{l}6 \text { out of } 6 \text { participants }(100 \%) \text { reported overal } \\
\text { improvement (excellent) }\end{array}$ \\
\hline Analgesics (non-narcotic) & Stable & Stable \\
\hline Narcotic analgesics & Stable & None \\
\hline Adverse events & None & $\begin{array}{l}\text { I participant reported shin splints, I reported } \\
\text { ankle pain, I reported knee pain }\end{array}$ \\
\hline
\end{tabular}




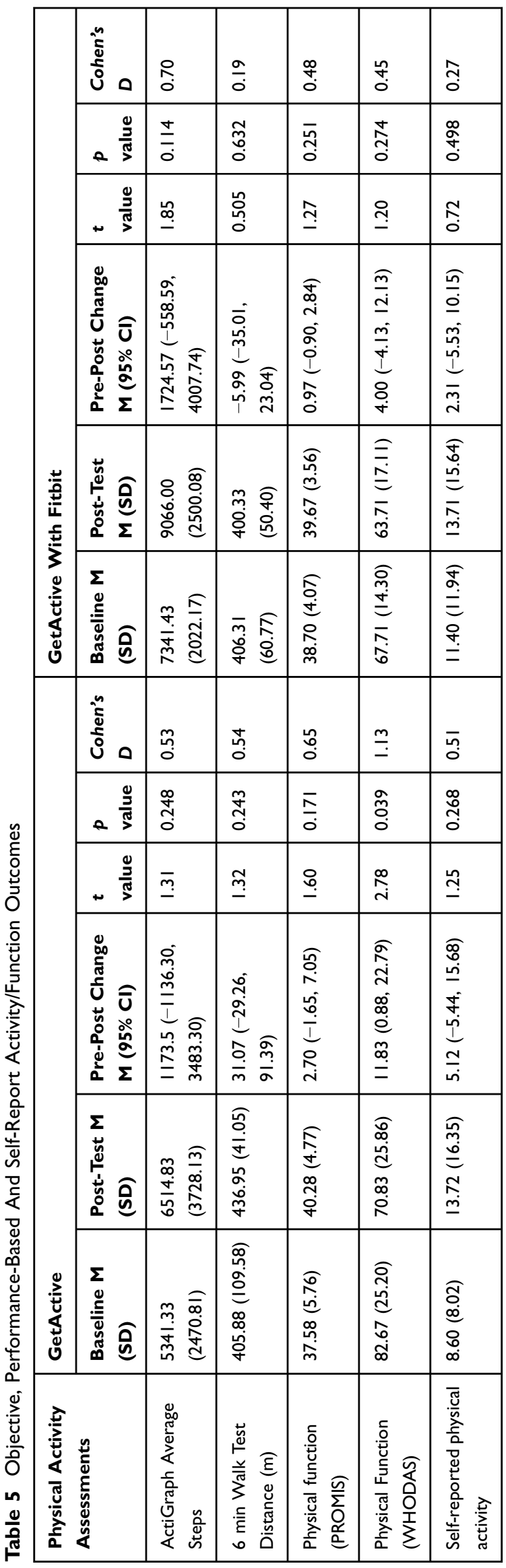

(>7300 steps, with most (5 out of 7) participants walking more than 5,000 steps a day). Improvements in number of steps in both groups were clinically meaningful, over the MCID of 1000 steps $^{43}$ (medium effect size). Four out of 7 participants within the GetActive with Fitbit group and 4 out of 6 within the GetActive group showed increases in step-count over the MCID (medium effect size).

Performance-based physical function. As a group, participants in the GetActive group generally increased their distance walked in the 6 min walk test (4 out of 6 participants; 1 above the MCID; medium effect size). Participants in the GetActive with Fitbit showed no change in the 6 min walk test (4 out of 7 participants increased distance but did not reach the MCID).

Self-report physical function. Both the GetActive and GetActive with Fitbit groups showed some improvement in physical function (medium effect size; Table 5).

\section{Emotional Function}

Both groups exhibited non-significant improvement in anxiety (small effect size) and depression (small to medium effect size; Table 6).

\section{Other Measures}

The GetActive group exhibited clinically meaningful reductions in levels of pain during rest and activity (medium effect size; Table 6), pain resilience (large effect size), and non-significant improvements in kinesiophobia (large effect size), and coping (medium-large effect size; Table 6). Participants in the GetActive with Fitbit group similarly exhibited a significant and clinically meaningful decrease in levels of pain during rest and activity (large effect sizes), as well as improvements in coping (large effect size), and a non-significant improvement in pain resilience (medium-large effect size). These comparisons were repeated using the non-parametric related-samples Wilcoxon signed rank test (see Analysis Plan) and yielded similar results in terms of direction and significance.

\section{Exit Interviews}

Participants reported high satisfaction with the learned relaxation, mind-body, and coping skills, and reported an improved ability to manage stress, pain, and an increased quality of life. They indicated that the pacing of the program, particularly in the first sessions, could be more relaxed and spread out over more sessions. Three participants indicated that healthy behaviors topics (e.g., healthy eating, sleep) detracted from the rest of the intervention. Participants in the GetActive 


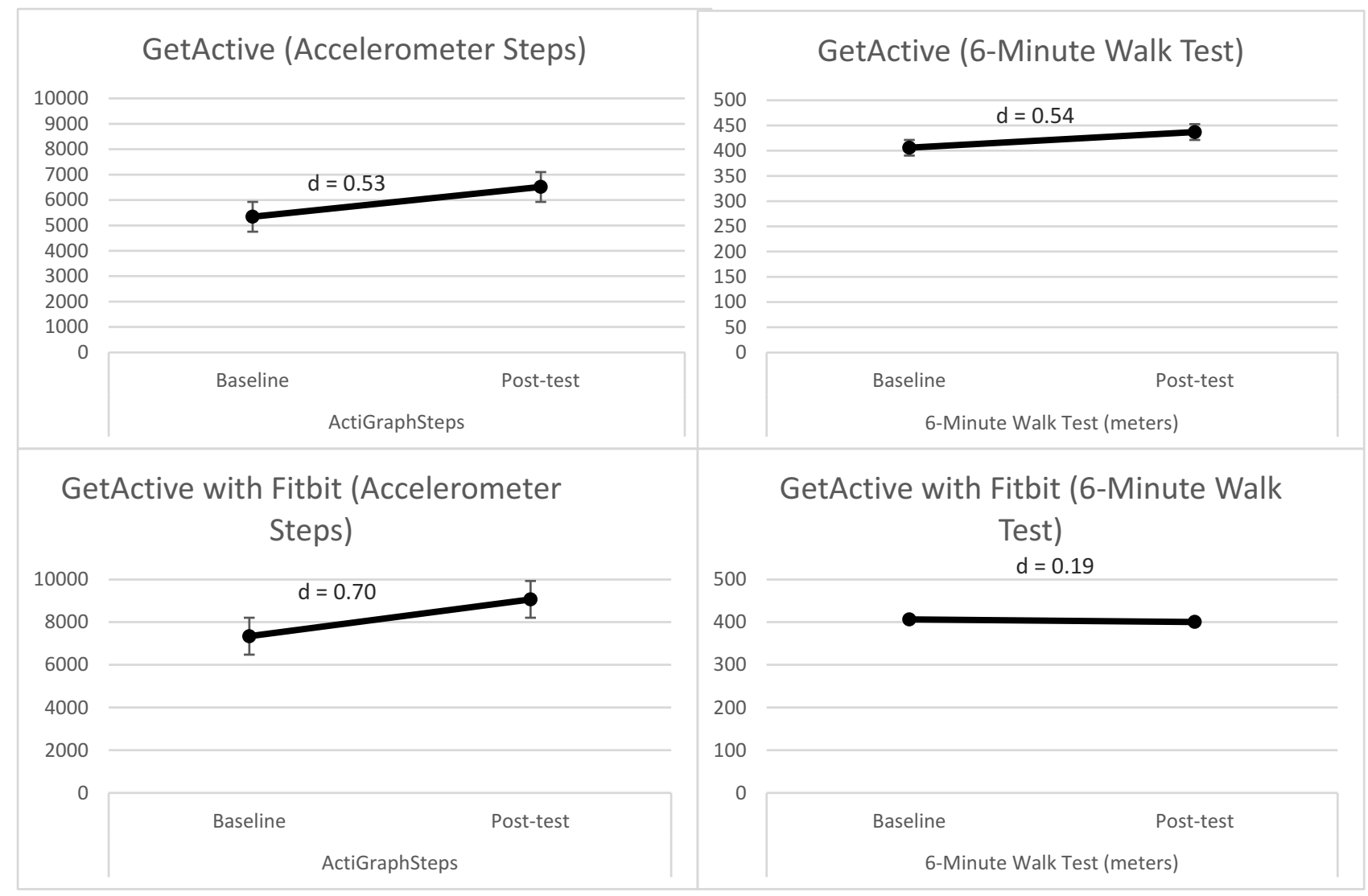

Figure 4 Pre-post changes in performance-based measures in the 2 programs.

with Fitbit group reported that the Fitbit was helpful in improving sleep and body awareness. However, they also reported feeling guilty and discouraged whenever they did not meet their step-goal or when receiving reminders about lack of activity.

\section{Discussion}

Despite decades of research, improvements in emotional and physical function following mind-body treatments among chronic pain patients are modest and generally fade over time. Further, no clinical trials to date assess physical function using IMMPACT and ICF criteria. Although there is evidence that physical activity helps patients with chronic pain, uptake and adherence remain problematic. The current study used the NIH stage model ${ }^{25}$ and NCCIH model for intervention development ${ }^{26}$ and adapted an evidence-based, group, mind-body program for the specific needs of patients with chronic pain. The study targeted comprehensive improvement in emotional and physical function ${ }^{9,10}$ (self-report, performance based and objective) with a Fitbit device (GetActive with Fitbit) or without it
(GetActive; phase 1). We also assessed preliminary feasibility markers for the two programs via 2 nonrandomized open pilot studies (phase 2).

Focus groups confirmed the physical, emotional, and social challenges associated with chronic pain. Fear of exacerbating pain, difficulties with pacing, and frustration with current functioning were common complaints from participants. They held largely favorable views of the modified 3RP and the additional pain management skills described, the use of a Fitbit device to increase activity, and the value of learning skills in a supportive group setting. The rationale of combining pain-specific mindbody skills with increased in walking linked to re-engagement in activities of daily living resonated with focus group participants. They identified the group setting and the Fitbit as particularly motivating in regards to meeting their goals. These results confirmed prior reports in this population. ${ }^{3,32}$ Results are also in line with evidence suggesting that this population is in need of extra support to engage in physical activity, ${ }^{62}$ and the essential roles of quota-based pacing and mind-body skills ${ }^{63}$ as aids in this endeavor. 


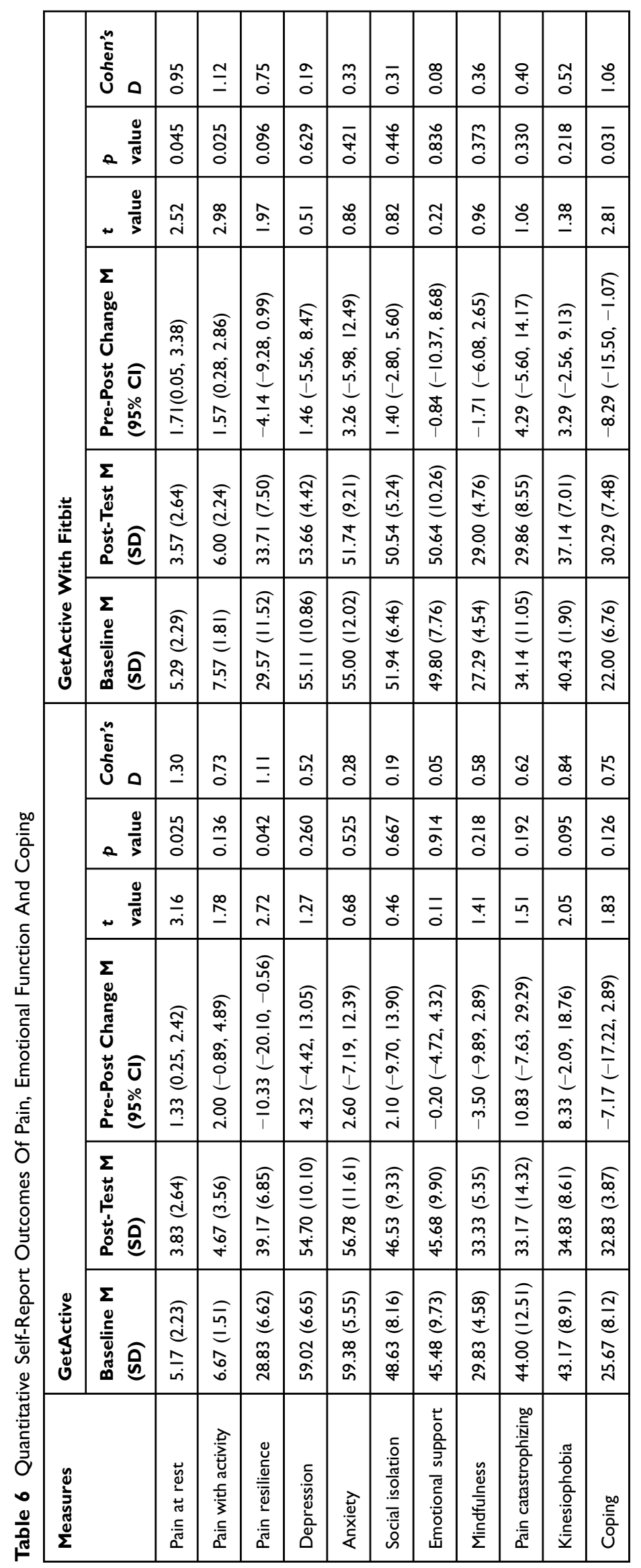


The open pilot studies showed excellent therapist adherence, client satisfaction, perceptions of improvement, feasibility of quantitative measures, and feasibility of recruitment for both programs. One hundred percent of participants with baseline data completed post-testing in both groups, and attrition was lower compared to other studies of chronic pain. ${ }^{4}$ Credibility and adherence to ActiGraph and Fitbit were high, and minimal adverse events were reported. Results indicate that the interventions and procedures are feasible and accepted by study participants.

In line with common guidelines for feasibility studies ${ }^{27}$ this study did not test efficacy or perform any betweengroup analyses. Nevertheless, preliminary exploration of effect sizes of within group pre-post changes suggests that both programs show promise for improvements in most outcome measures. Critically, both groups exhibited stepcount increases that are clinically meaningful. ${ }^{43}$ Moreover, we found large effect sizes and clinically meaningful ${ }^{54}$ decreases in pain following the programs, as well as large and significant effects for improvements in physical function and medium-large effect sizes for improvements in coping, pain resilience, and kinesiophobia.

Qualitative exit interviews indicated high satisfaction with both programs and the mind-body, pain-specific and physical activity skills learned, and suggested additional modifications including: 1) increasing the total number of sessions in both programs from 8 to 10 to allow for a more relaxed pace of teaching the skills; 2) including 3RP specific information on healthy eating and sleep in an appendix to avoid deterring from the flow of the program; 3 ) debunking the myths regarding mindfulness, meditation, and other mind-body practices which had associations with "laziness" for some participants; 4) incorporating a more thorough discussion on challenges associated with forming new habits, as well as introducing the skill of self-compassion to help patients remain motivated even when not meeting step-count goals; 5) developing a comprehensive assessment of activity (e.g., job activity, seating time, Physical Activity Questionnaire-Short Form IPAQ-SF ${ }^{64}$ scores) during screening to ensure that participants are sedentary (e.g., less than 5,000 steps) so that they can fully benefit from the programs.

\section{Conclusions}

This study is the first to integrate physical activity with mindbody skills to increase comprehensive physical and emotional functioning in a heterogeneous group of patients with chronic pain, the first to use a comprehensive assessment of physical function consistent with IMMPACT and ICF criteria, and the first to compare increase in activity with and without a Fitbit. Focus groups, open pilot, and exit interview data provided preliminary evidence for feasibility and acceptability of the novel interventions as well as a signal of improvement in physical activity, pain, physical function, and other measures. Consistent with the aims of pilot feasibility studies, ${ }^{27}$ our aim was not to examine the efficacy of the programs or generalize findings to the larger pain population. Rather, we collected meaningful information which allowed us to further modify the GetActive with Fitbit and GetActive programs and study procedures which are currently being tested within a definitive feasibility pilot RCT, following the NIH stage model ${ }^{25}$ and $\mathrm{NCCIH}$ framework. ${ }^{26}$ If feasibility benchmarks are met within this feasibility RCT of GetActive versus GetActive with Fitbit, we will next conduct a fully powered RCT of the GetActive with Fitbit versus Get Active versus a health education control in order to determine comparatively their efficacy and sustained improvement in emotional and physical function, as well as elucidate whether the Fitbit is a necessary ingredient. Results of the future efficacy RCT will have important implications for the treatment of chronic pain and may lead to a paradigm shift in the manner in which pain is assessed and treated, support implementation of ICF and IMMPACT criteria across all settings, and potentially provide evidence for the use of digital activity trackers as activity reinforcers within chronic pain treatments.

\section{Acknowledgement}

We thank Stephanie Moffat and Christopher Funes for transcribing the interviews.

\section{Funding}

This study was funded by an R34 grant from the National Center for Complementary and Integrative Health (1R34AT009356-01A1) to the senior author.

\section{Disclosure}

Dr. Schatman serves as a consultant to Kaleo Pharma and Quest Diagnostics and received ad board honorarium from Salix Pharmaceuticals. All other authors declare that they have no conflicts of interest in this work.

\section{References}

1. IASP Taxonomy Working Group. Pain terms a current list with definitions and notes on usage. Pain. 1986;4:S215-S221. doi:10.1016/03043959(86)90113-2 
2. Schappert SM. Ambulatory care visits to physician offices, hospital outpatient departments, and emergency departments: United States, 2001-02. Vital Health Stat. 2006;13.

3. Vranceanu AM, Bachoura A, Weening A, Vrahas M, Smith RM, Ring D. Psychological factors predict disability and pain intensity after skeletal trauma. J Bone Jt Surg - Ser A. 2014;96(3). doi:10.2106/JBJS. L.00479

4. Veehof MM, Oskam MJ, Schreurs KMG, Bohlmeijer ET. Acceptance-based interventions for the treatment of chronic pain: a systematic review and meta-analysis. Pain. 2011;152(3):533-542. doi:10.1016/j.pain.2010.11.002

5. Gotink RA, Chu P, Busschbach JJV, Benson H, Fricchione GL, Hunink MGM. Standardised mindfulness-based interventions in healthcare: an overview of systematic reviews and meta-analyses of RCTs. PLoS One. 2015;10(4). doi:10.1371/journal.pone.0124344

6. Hilton L, Hempel S, Ewing BA, et al. Mindfulness meditation for chronic pain: systematic review and meta-analysis. Ann Behav Med. 2017;51(2):199-213. doi:10.1007/s12160-016-9844-2

7. Jackson W, Kulich R, Malacarne A, Lapidow A, Vranceanu A. Physical functioning and mindfulness based interventions in chronic pain: a systematic review. $J$ Pain. 2016;17:S99. doi:10.1016/j. jpain.2016.01.304

8. Marikar Bawa FL, Mercer SW, Atherton RJ, et al. Does mindfulness improve outcomes in patients with chronic pain? Systematic review and meta-analysis. $\mathrm{Br} J$ Gen Pract. 2015;65(635):e387-e400. doi:10.3399/bjgp15X685297

9. Taylor AM, Phillips K, Patel KV, et al. Assessment of physical function and participation in chronic pain clinical trials: IMMPACT/OMERACT recommendations. Pain. 2016;157(9):1836-1850. doi:10.1097/j.pain. 0000000000000577

10. World Health Organization. International Classification of Functioning, Disability and Health (ICF). Geneva; 2001.

11. Jackson W, Zale EL, Berman SJ, et al. Physical functioning and mindfulness skills training in chronic pain: a systematic review. $J$ Pain Res. 2019;12:179-189. doi:10.2147/JPR

12. Geneen LJ, Moore RA, Clarke C, Martin D, Colvin LA, Smith BH. Physical activity and exercise for chronic pain in adults: an overview of cochrane reviews. Cochrane Database Syst Rev. 2017;1: CD011279. doi:10.1002/14651858.CD011279.pub2

13. Endrighi R, Steptoe A, Hamer M. The effect of experimentally induced sedentariness on mood and psychobiological responses to mental stress. Br J Psychiatry. 2016;208(3):245-251. doi:10.1192/ bjp.bp. 114.150755

14. Suchert V, Hanewinkel R, Isensee B. Sedentary behavior and indicators of mental health in school-aged children and adolescents: a systematic review. Prev Med (Baltim). 2015;76:48-57. doi:10.1016/ j.ypmed.2015.03.026

15. O'Connor SR, Tully MA, Ryan B, et al. Walking exercise for chronic musculoskeletal pain: systematic review and meta-analysis. Arch Phys Med Rehabil. 2015;96(4):724-734.E3. doi:10.1016/j. apmr.2014.12.003

16. Kent $P$, Kjaer P. The efficacy of targeted interventions for modifiable psychosocial risk factors of persistent nonspecific low back pain - a systematic review. Man Ther. 2012;17(5):385-401. doi:10.1016/j. math.2012.02.008

17. Rainville J, Hartigan C, Martinez E, Limke J, Jouve C, Finno M. Exercise as a treatment for chronic low back pain. Spine J. 2004;4 (1):106-115. doi:10.1016/S1529-9430(03)00174-8

18. Vranceanu A-M, Stone M, Wallace T, Kulich R. Cognitive behavioral therapy for chronic pain. In: Vranceanu A-M, Greer J, Safren S editors. The Massachusetts General Hospital Handbook of Behavioral Medicine. Humana Press; 2017:93-114. doi:10.1007/ 978-3-319-29294-6_5

19. Nijs J, Kosek E, Van Oosterwijck J, Meeus M. Dysfunctional endogenous analgesia during exercise in patients with chronic pain: to exercise or not to exercise? Pain Physician. 2012;15:ES205-ES213.
20. Patel MS, Asch DA, Volpp KG. Wearable devices as facilitators, not drivers, of health behavior change. JAMA. 2015;313(5):459-460. doi:10.1001/jama.2014.14781

21. Chandrasekar A, Hensor EMA, Mackie SL, Backhouse MR, Harris E. Preliminary concurrent validity of the fitbit-zip and actigraph activity monitors for measuring steps in people with polymyalgia rheumatica. Gait Posture. 2018;61:339-345. doi:10.1016/j. gaitpost.2018.01.035

22. Alharbi M, Bauman A, Neubeck L, Gallagher R. Measuring overall physical activity for cardiac rehabilitation participants: a review of the literature. Hear Lung Circ. 2017;26(10):1008-1025. doi:10.1016/ j.hlc.2017.01.005

23. Gordon R, Bloxham S. Influence of the Fitbit Charge HR on physical activity, aerobic fitness and disability in non-specific back pain participants. J Sport Med Phys Fit. 2017. 57(12):1669-1675.

24. Jamison RN, Jurcik DC, Edwards RR, Huang CC, Ross EL, Pilot A. Comparison of a smartphone app with or without 2-way messaging among chronic pain patients: who benefits from a pain app? Clin $J$ Pain. 2017;33(8):676-686. doi:10.1097/AJP.0000000000000455

25. Onken LS, Carroll KM, Shoham V, Cuthbert BN, Riddle M. Reenvisioning clinical science: unifying the discipline to improve the public health. Clin Psychol Sci. 2013;2(1):22-34. doi:10.1177/ 2167702613497932

26. National Center for Complementary and Integrative Health. Framework for developing and testing mind and body interventions. Available from: https://nccih.nih.gov/grants/mindbody/framework. Published 2017. Accessed June 27, 2019.

27. Leon AC, Davis LL, Kraemer HC. The role and interpretation of pilot studies in clinical research. J Psychiatr Res. 2011;45(5):626-629. doi:10.1016/j.jpsychires.2010.10.008

28. Lancaster GA, Dodd S, Williamson PR. Design and analysis of pilot studies: recommendations for good practice. $J$ Eval Clin Pract. 2004;10(2):307-312. doi:10.1111/j.2002.384.doc.x

29. Park ER, Traeger L, Vranceanu AM, et al. The development of a patient-centered program based on the relaxation response: the relaxation response Resiliency Program (3RP). Psychosomatics. 2013;54(2):165-174. doi:10.1016/j.psym.2012.09.001

30. Gonzalez A, Shim M, Mahaffey B, Vranceanu A-M, Reffi A, Park ER. The Relaxation Response Resiliency Program (3RP) in patients with headache and musculoskeletal pain: a retrospective analysis of clinical data. Pain Manag Nurs. 2018. doi:10.1016/j.pmn.2018.04.003

31. Eisenberg DM, Buring JE, Hrbek AL, et al. A model of integrative care for low-back pain. J Altern Complement Med. 2012;18(4):354362. doi: $10.1089 / \mathrm{acm} .2011 .0408$

32. Crombez G, Eccleston C, Van Damme S, Vlaeyen JWS, Karoly P. Fear-avoidance model of chronic pain: the next generation. Clin J Pain. 2012;28(6):475-483. doi:10.1097/AJP.0b013e3182385392

33. Breivik H, Collett B, Ventafridda V, Cohen R, Gallacher D. Survey of chronic pain in Europe: prevalence, impact on daily life, and treatment. Eur J Pain. 2006;10(4):287-333. doi:10.1016/j.ejpain.2005.06.009

34. Dahl J. Behavioral activation for depression: a clinician's guide. Cogn Behav Ther. 2011;40(1):77. doi:10.1080/16506073.2010.514119

35. American Thoractic Society. ATS statement: the six-minute walk test. Am J Respir Crit Care Med. 2002;166(1):111-117. doi:10.1164/ rccm.166/1/111

36. Ritchie J, Lewis J, McNaughton Nicholls C, Ormston R. Qualitative Research Practice: A Giode for Social Science Students and Researchers. 2nd ed ed. Sage Publications, Inc; 2013.

37. Tong A, Sainsbury P, Craig J. Consolidated criteria for reporting qualitative research (COREQ): A 32-item checklist for interviews and focus groups. Int J Qual Heal Care. 2007;19(6):349-357. doi:10.1093/intqhe/mzm042

38. Smith J, Firth J. Qualitative data analysis: the framework approach. Nurse Res. 2011. doi:10.7748/nr2011.01.18.2.52.c8284

39. Bazeley PK. Qualitative Data Analysis with NVivo. Los Angeles: Sage; 2013. 
40. Rounsaville BJ, Carroll KM, Onken LS, Stage A. Model of behavioral therapies research: getting started and moving on from stage I. Clin Psychol Sci Pract. 2006;8(2):133-142. doi:10.1093/clipsy.8.2.133

41. Czajkowski SM, Powell LH, Adler N, et al. From ideas to efficacy: the ORBIT model for developing behavioral treatments for chronic diseases. Heal Psychol. 2015;34(10):971-982. doi:10.1037/hea0000161

42. Attkisson CC, Zwick R. The client satisfaction questionnaire. Psychometric properties and correlations with service utilization and psychotherapy outcome. Eval Program Plann. 1982;5(3):233237. doi:10.1016/0149-7189(82)90074-X

43. Marshall SJ, Levy SS, Tudor-Locke CE, et al. Translating physical activity recommendations into a pedometer-based step goal. 3000 steps in 30 mins. Am $J$ Prev Med. 2009;36(5):410-415. doi:10.1016/j.amepre.2009.01.021

44. Redelmeier DA, Bayoumi AM, Goldstein RS, Guyatt GH. Interpreting small differences in functional status: the six minute walk test in chronic lung disease patients. Am J Respir Crit Care Med. 1997;155(4):1278-1282. doi:10.1164/ajrccm.155.4.9105067

45. Stone AA, Broderick JE, Junghaenel DU, Schneider S, Schwartz JE. PROMIS fatigue, pain intensity, pain interference, pain behavior, physical function, depression, anxiety, and anger scales demonstrate ecological validity. J Clin Epidemiol. 2016;74:194-206. doi:10.1016/ j.jclinepi.2015.08.029

46. Hung MM, Saltzman CL, Bounsanga J, et al. What Are the MCIDs for PROMIS, NDI, and ODI instruments among patients with spinal conditions? Clin Orthop Relat Res. 2018;476(10):2027-2036. doi:10.1097/CORR.0000000000000419

47. World Health Organization. Whodas 2.0. Assessment. 2010.

48. Washburn RA, Zhu W, McAuley E, Frogley M, Figoni SF. The physical activity scale for individuals with physical disabilities: development and evaluation. Arch Phys Med Rehabil. 2002;83 (2):193-200. doi:10.1053/apmr.2002.27467

49. A brief guide to the PROMIS depression instruments. Patient reported outcomes measurement information system. Available from: https://www.assessmentcenter.net/documents/PROMIS Depression Scoring Manual.pdf. Published 2015. Accessed October 29, 2018.

50. A brief guide to the PROMIS anxiety instruments. Outcomes measurement information system. Available from: https://www.assess mentcenter.net/documents/PROMIS Anxiety Scoring Manual.pdf. Published 2015. Accessed October 29, 2018.

51. Selvarajah S, Neuman BJ, Skolasky RL. Using PROMIS health domains to identify clinically meaningful change in lumbar degenerative spine disease: concurrent validity and responsiveness. Spine J. 2016;16(10):S369-S370. doi:10.1016/j.spinee.2016.07.304

52. Rodriguez CS. Pain measurement in the elderly: A review. Pain Manag Nurs. 2001;2(2):38-46. doi:10.1053/jpmn.2001.23746
53. Farrar JT, Young JP, LaMoreaux L, Werth JL, Poole RM. Clinical importance of changes in chronic pain intensity measured on an 11point numerical pain rating scale. Pain. 2001;94(2):149-158. doi:10.1016/S0304-3959(01)00349-9

54. Salaffi F, Stancati A, Alberto Silvestri C, Ciapetti A, Grassi W. Minimal clinically important changes in chronic musculoskeletal pain intensity measured on a numerical rating scale. Eur J Pain. 2004;8(4):283-291. doi:10.1016/j.ejpain.2003.09.004

55. A brief guide to the PROMIS Social Isolation instruments. Outcomes measurement information system. Available from: http://www.health measures.net/administrator/components/com_instruments/uploads/ 15-09-01 16-44-48 PROMISSocialIsolationScoringManual.pdf. Published 2015. Accessed October 29, 2018.

56. Feldman G, Hayes A, Kumar S, Greeson J, Laurenceau JP. Mindfulness and emotion regulation: the development and initial validation of the Cognitive and Affective Mindfulness ScaleRevised (CAMS-R). J Psychopathol Behav Assess. 2007;29(3):177190. doi:10.1007/s10862-006-9035-8

57. A brief guide to the PROMIS Emotional Support instruments. Outcomes measurement information system. Available from: https:// www.assessmentcenter.net/documents/PROMIS Emotional Support Scoring Manual.pdf. Published 2015. Accessed October 29, 2018.

58. Sullivan MJL, Bishop SR, Pivik J. The pain catastrophizing scale: development and validation. Psychol Assess. 1995;7(4):524-532. doi:10.1037/1040-3590.7.4.524

59. Woby SR, Roach NK, Urmston M, Watson PJ. Psychometric properties of the TSK-11: A shortened version of the Tampa Scale for Kinesiophobia. Pain. 2005;117(1-2):137-144. doi:10.1016/j.pain.2005.05.029

60. Carver CS MOCS (Measure of Current Status). Available from: http://www.psy.miami.edu/faculty/ccarver/sclMOCS.html. Published 2006. Accessed June 19, 2019.

61. Geisser ME, Clauw DJ, Strand V, Gendreau RM, Palmer R, Williams DA. Contributions of change in clinical status parameters to Patient Global Impression of Change (PGIC) scores among persons with fibromyalgia treated with milnacipran. Pain. 2010;149(2):373-378. doi:10.1016/j.pain.2010.02.043

62. Joelsson M, Bernhardsson S, Larsson MEH. Patients with chronic pain may need extra support when prescribed physical activity in primary care: a qualitative study. Scand J Prim Health Care. 2017;35 (1):64-74. doi:10.1080/02813432.2017.1288815

63. Nielson WR, Jensen MP, Karsdorp PA, Vlaeyen JWS. Activity pacing in chronic pain: concepts, evidence, and future directions. Clin J Pain. 2013;29(5):461-468. doi:10.1097/AJP.0b013e3182608561

64. Lee PH, Macfarlane DJ, Lam TH, Stewart SM. Validity of the international physical activity questionnaire short form (IPAQ-SF): A systematic review. Int J Behav Nutr Phys Act. 2011;8. doi:10.1186/ 1479-5868-8-115
Journal of Pain Research

\section{Publish your work in this journal}

The Journal of Pain Research is an international, peer reviewed, open access, online journal that welcomes laboratory and clinical findings in the fields of pain research and the prevention and management of pain. Original research, reviews, symposium reports, hypothesis formation and commentaries are all considered for publication. The manuscript

Submit your manuscript here: https://www.dovepress.com/journal-of-pain-research-journa management system is completely online and includes a very quick and fair peer-review system, which is all easy to use. Visit http:// www.dovepress.com/testimonials.php to read real quotes from published authors. 